\title{
A 60-GHz interferometer with a local oscillator integrated antenna array for divertor simulation experiments on GAMMA 10/PDX
}

J. Kohagura, M. Yoshikawa, X. Wang, D. Kuwahara, N. Ito, Y. Nagayama, Y. Shima, K. Nojiri, M. Sakamoto, Y. Nakashima, and A. Mase

Citation: Rev. Sci. Instrum. 87, 11E127 (2016); doi: 10.1063/1.4961292

View online: http://dx.doi.org/10.1063/1.4961292

View Table of Contents: http://aip.scitation.org/toc/rsi/87/11

Published by the American Institute of Physics

\section{Ф SHIMADZU Powerful, Multi-functional UV-Vis-NIR and}

Excellence in Science FJus Spectsophotometers

Providing the utmost in sensitivity, accuracy and resolution for applications in materials characterization and science

- Photovoltaics

- Polymers

- Coatings

- Paints

Click here for accurate, cost-effective laboratory solutions
- DNA film structures

- Packaging materials

- Nanotechnology

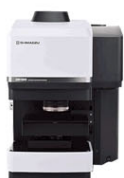

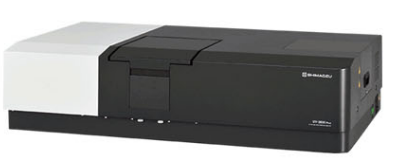

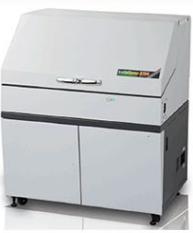




\title{
A 60-GHz interferometer with a local oscillator integrated antenna array for divertor simulation experiments on GAMMA 10/PDX
}

\author{
J. Kohagura, ${ }^{1, a)}$ M. Yoshikawa, ${ }^{1}$ X. Wang, ${ }^{2}$ D. Kuwahara,${ }^{3}$ N. Ito, ${ }^{4}$ Y. Nagayama,${ }^{5}$ Y. Shima,${ }^{1}$ \\ K. Nojiri, ${ }^{1}$ M. Sakamoto, ${ }^{1}$ Y. Nakashima, ${ }^{1}$ and A. Mase ${ }^{6}$ \\ ${ }^{1}$ Plasma Research Center, University of Tsukuba, Tsukuba, Ibaraki 305-8577, Japan \\ ${ }^{2}$ Saitama University, Saitama 338-8570, Japan \\ ${ }^{3}$ Tokyo University of Agriculture and Technology, Koganei, Tokyo 184-8588, Japan \\ ${ }^{4}$ National Institute of Technology, Ube College, Ube, Yamaguchi 755-8555, Japan \\ ${ }^{5}$ National Institute of Fusion Science, Toki, Gifu 509-5292, Japan \\ ${ }^{6}$ Kyushu University, Kasuga, Fukuoka 816-8580, Japan
}

(Presented 7 June 2016; received 9 June 2016; accepted 5 August 2016; published online 31 August 2016)

\begin{abstract}
In conventional multichannel/imaging microwave diagnostics of interferometry, reflectometry, and electron cyclotron emission measurements, a local oscillator (LO) signal is commonly supplied to a receiver array via irradiation using LO optics. In this work, we present a $60-\mathrm{GHz}$ interferometer with a new eight-channel receiver array, called a local oscillator integrated antenna array (LIA). An outstanding feature of LIA is that it incorporates a frequency quadrupler integrated circuit for LO supply to each channel. This enables simple and uniform LO supply to the receiver array using only a 15-GHz LO source and a coaxial cable transmission line instead of using an expensive $60-\mathrm{GHz}$ source, $\mathrm{LO}$ optics, and a waveguide transmission line. The new interferometer system is first applied to measure electron line-averaged density inside the divertor simulation experimental module (D-module) on GAMMA 10/PDX tandem mirror device. Published by AIP Publishing. [http://dx.doi.org/10.1063/1.4961292]
\end{abstract}

\section{INTRODUCTION}

Highly spatially and temporally resolved electron density profile and its fluctuation behavior are necessary in order to solve many of important physics issues in magnetized plasmas. Multichannel/imaging microwave diagnostics ${ }^{1}$ are powerful tools to obtain spatially and temporally resolved such key parameters. In microwave diagnostics such as interferometry, reflectometry, and electron cyclotron emission measurements, a local oscillator (LO) signal is often used to convert radio frequency (RF) signal into an intermediate frequency (IF) signal. ${ }^{2}$ In these diagnostics using one- or two-dimensional receiver array, LO signal is commonly supplied to a receiver array by irradiation using LO optics and a high-power LO source. ${ }^{1}$ However, the conversion efficiency of each receiver channel can be affected by non-uniform irradiation pattern. ${ }^{3}$

Recently a new receiver array has been developed to overcome the disadvantage. That is a local oscillator integrated antenna array (LIA). ${ }^{4,5}$ One of the unique features of LIA is that it includes a frequency multiplier for LO supply to each channel and due to this no high-frequency LO irradiation is required. In this work, we present a new interferometer system with LIA. The system was applied to measure the electron line density in divertor simulation experiments in GAMMA 10/PDX device. ${ }^{6,7}$

\footnotetext{
Note: Contributed paper, published as part of the Proceedings of the 21st Topical Conference on High-Temperature Plasma Diagnostics, Madison, Wisconsin, USA, June 2016.

a) Author to whom correspondence should be addressed. Electronic mail: kohagura@prc.tsukuba.ac.jp.
}

\section{EXPERIMENTAL APPARATUS}

GAMMA $10 / \mathrm{PDX}^{6,7}$ is a 27 -m-long tandem mirror machine (Fig. 1). In standard hot-ion-mode operation ion cyclotron range of frequency (ICRF) waves called RF1 and RF2 are injected for the MHD stabilization at the anchor cells and for ion heating in the central cell, respectively. High heat and particle end loss fluxes are produced at the end mirror exit of GAMMA 10/PDX. ${ }^{6,7}$

A divertor simulation experimental module called D-module has been installed at the west end region for divertor simulation study. D-module is a rectangular chamber with an inlet aperture. Two tungsten target plates $\left(350 \times 300 \mathrm{~mm}^{2}\right)$ are mounted in V-shape inside D-module. Gas injection lines are installed to study radiation cooling and plasma detachment. Thirteen Langmuir probes installed on one of the target plates are used to obtain local electron temperatures and densities on the target. However, no information on electron density in the internal space between the target plates had been obtained. To measure electron density inside D-module, an interferometer system was developed and utilized.

\section{NEW 60-GHZ INTERFEROMETER WITH LIA}

\section{A. Previous interferometer system}

The previous interferometer system ${ }^{8,9}$ is briefly summarized as follows: A horn-antenna mixer array (HMA) $)^{10,11}$ originally designed for microwave imaging reflectometry on the large helical device (LHD) was used as a receiver array. The one-dimensional seven-channel HMA is made by 


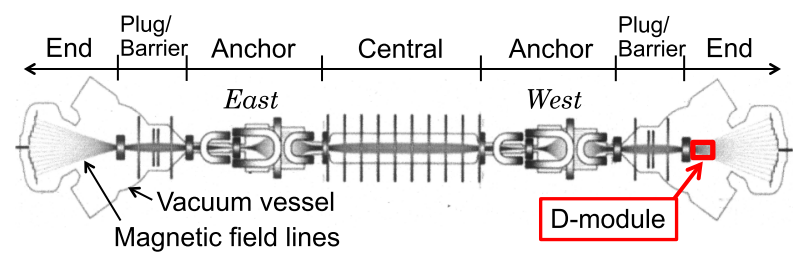

FIG. 1. Top view of GAMMA 10/PDX and D-module.

holding a printed circuit board (PCB) between an upper and a lower frame with half apertures of pyramidal horn antennas. Schottky barrier diodes are mounted on the PCB as mixers. Since the $\mathrm{LO}$ wave $(60 \mathrm{GHz})$ irradiation is needed for HMA to convert RF wave $(60.15 \mathrm{GHz})$ from plasma into IF signal $(150 \mathrm{MHz})$, the system requires a high-cost high-power $60 \mathrm{GHz}$ LO source system, a LO antenna with a waveguide transmission line, and a beam splitter.

\section{B. Interferometer system with LIA}

An eight-channel LIA ${ }^{4,5}$ consists mainly of horn antennas, waveguide to microstrip line transition (WMT), mixers, LO modules, LO power divider, and IF transmission line. The size of LIA is $227 \times 184 \times 20 \mathrm{~mm}^{3}$. The horn aperture size is $19.5 \times 19.5 \mathrm{~mm}^{2}$, and the channel distance is $20 \mathrm{~mm}$. An outstanding feature of LIA is that it incorporates a monolithic microwave integrated circuit (MMIC) quadrupler for LO supply in each channel element. The pre-LO, that is, the $1 / 4$ frequency LO power input to LIA is divided into eight ways by a Wilkinson power divider circuit. The pre-LO is then frequency multiplied up to $60 \mathrm{GHz}$ by the MMIC quadrupler on the PCB. Mixing RF signal from plasma and the LO signal is realized using microstrip line on the circuit board. This enables simple and uniform LO supply to each channel of LIA by only a $15-\mathrm{GHz}$ LO source and a coaxial cable transmission line instead of using a $60-\mathrm{GHz}$ source, $\mathrm{LO}$ optics, and a waveguide transmission line.

The new interferometer system with LIA is shown in Figs. 2 and 3. The system is based on a frequency-multiplied heterodyne scheme using two oscillators. A $15 \mathrm{GHz} \mathrm{LO}$ wave generated by the phase-locked dielectric resonator oscillator (PLDRO) is up-converted to $15.0375 \mathrm{GHz}$ by mixing a 37.5 MHz signal from a temperature-compensated crystal oscillator (TCXO). This $1 / 4$ frequency RF wave is frequency multiplied to $60.15 \mathrm{GHz}$ by a quadrupler. The RF wave emitted from the antenna is converted to the parallel beam

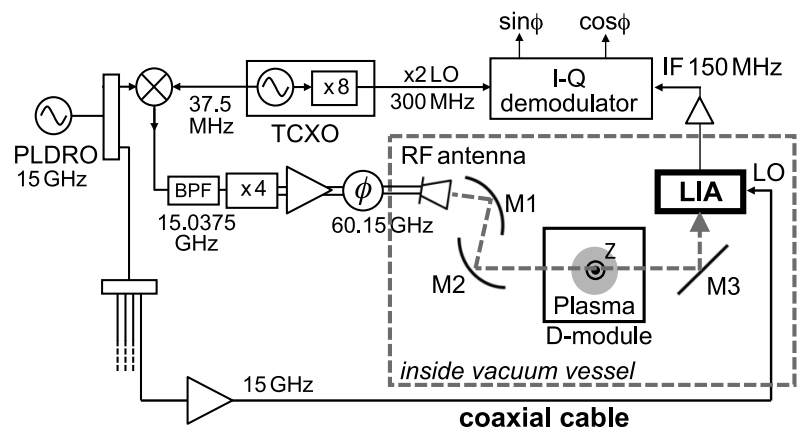

FIG. 2. Newly developed interferometer system using LIA.

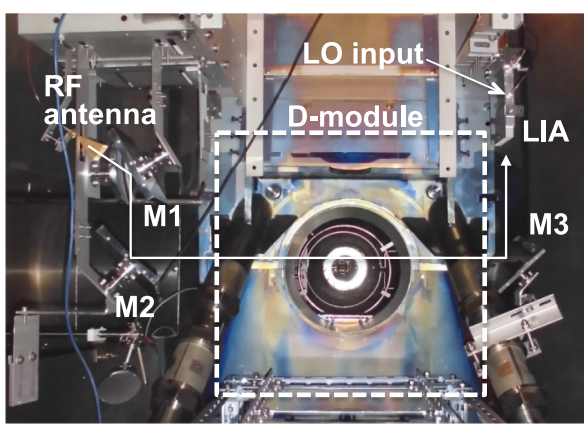

FIG. 3. Interferometer system (D-module is in a down-position).

of $\sim 150 \mathrm{~mm}$ in diameter by two mirrors. In the former system, the $1 / 4$ frequency $L O$ wave was first frequency multiplied up to $60 \mathrm{GHz}$. Then it was amplified and transmitted via waveguide to a LO antenna. A beam splitter was used to combine the $\mathrm{RF}$ wave and the LO wave. In the new system, a $15 \mathrm{GHz}$ LO signal is simply amplified and transmitted directly to LIA using a coaxial semirigid cable line. The merits of the system are as follows: (i) The expensive quadrupler and amplifier at V-band are replaced by the cheaper Ku-band amplifier. The MMIC quadrupler used on the LIA circuit is much cheaper than commercially available waveguide quadrupler module. (ii) Uniform, direct LO supply is easily achieved. (iii) No power loss of RF signal by a beam splitter occurs.

Shown in Fig. 4 are the schematic (a) top view and (b) side view of $\mathrm{D}$-module with the $\mathrm{V}$-shaped target plates at open angle $45^{\circ}$. Measurement position of the interferometer is indicated by the arrow in Fig. 4(a) and the position where the viewing chord intersects with the Z-axis is marked as the solid short line in Fig. 4(b). Microwave is launched from a horizontal viewing port of D-module at an angle of $65^{\circ}$ against the Z-axis due to the limitation of space and viewing port size. Only three channels of LIA aligned in the $\mathrm{Z}$ direction are available due to the same reason. The center of the measured position on the $\mathrm{Z}$ axis is $-224,-244$, and $-264 \mathrm{~mm}$ (when the location of the target plates corner is defined as $Z=0$ ). The length of the interferometer chords across the plasma in D-module is $\sim 26 \mathrm{~cm}$. This value is used to evaluate a line-averaged density from a line-integrated density. Magnetic field lines and positions of the probes (closed circles) are also illustrated. Magnetic field strength is about 0.2-0.3 $\mathrm{T}$ along the lines of sight. Although only one array of LIA is used in the present system, a $2 \mathrm{D}$ receiver can easily be made by stacking the several pieces of LIAs, which enables a 2D density distribution measurement. (a)

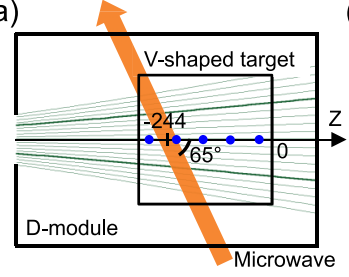

(b)

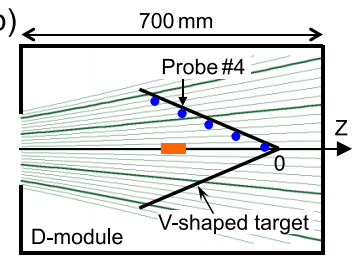

FIG. 4. (a) Top and (b) side views of D-module. 

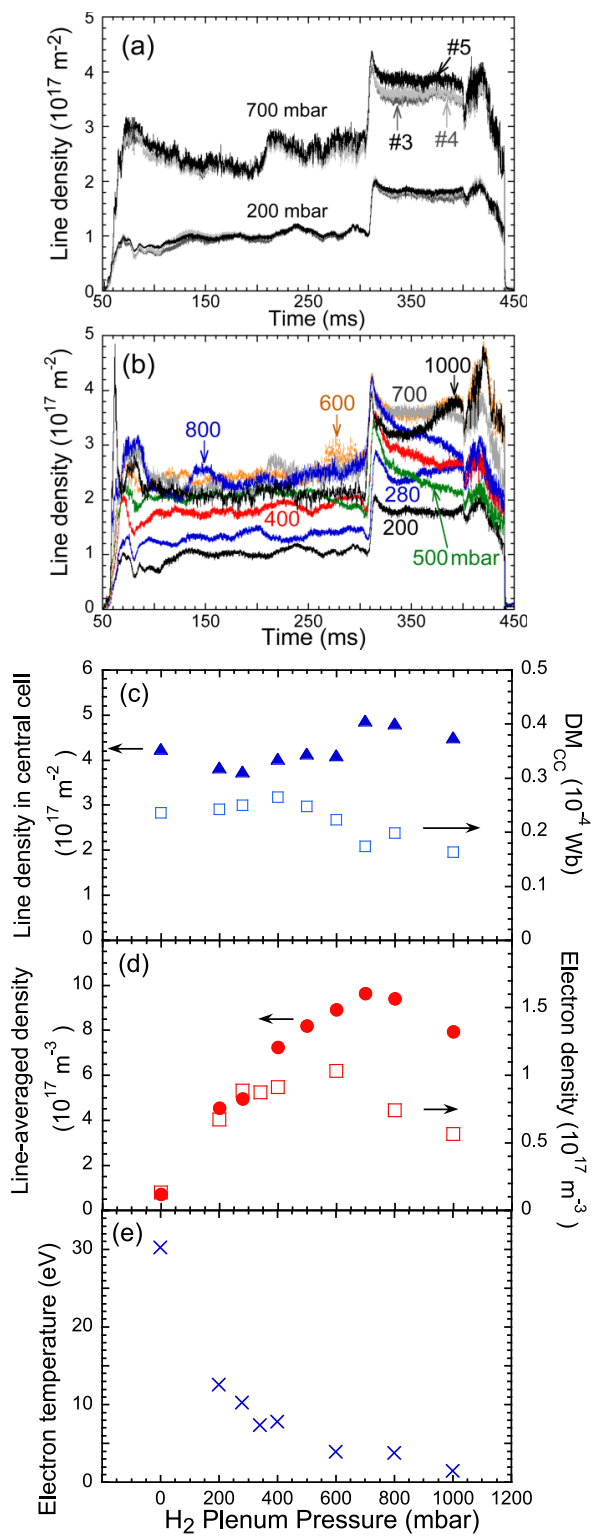

FIG. 5. Time behaviors of (a) the line densities measured by the three interferometer codes, (b) the line density of chord No. 4 with different plenum pressures. The plenum pressure dependence of (c) the line density and diamagnetism in the central cell, (d) the line-averaged densities obtained by the interferometer and the probe, and (e) the electron temperature on the target plate.

\section{EXPERIMENTAL RESULTS}

The $\mathrm{H}_{2}$ gas was injected in D-module at $0.3 \mathrm{~s}$ before the plasma discharge with 0.75 -s duration. The plenum pressure of a gas reservoir was set from 200 to 1000 mbar. Standard hot-ion mode plasma was produced by RF1 and RF2 at the upstream region of the central cell. The additional heating of RF3 was applied during $t=300-400 \mathrm{~ms}$ at the east anchor region. In Fig. 5(a), the time behaviors of line densities inside the $\mathrm{V}$-shaped target measured by the interferometer are shown at 200 and 700 mbar through three chords (Nos. 3-5), respectively. In this experiment, not much difference is observed among the densities of the three channels especially before applying RF3. Figure 5(b) shows the line densities through the chord No. 4 with different pressures.

Figures 5(c)-5(e) show several plasma parameters during RF1 and RF2 period as a function of $\mathrm{H}_{2}$ gas plenum pressure. The plenum pressure dependence of the electron line density and diamagnetism measured at the central cell is presented in Fig. 5(c). The gas injection in D-module affects the plasma production at the upstream region. Roughly speaking, the stored energy decreases and the electron density increases with the increase of gas pressure from the range of 200-600 mbar to the range of 700-1000 mbar. Figure 5(d) shows the lineaveraged density measured by the interferometer (the closed circle) and the electron density measured by the Langmuir probe \#4 on the target plate (the open square), respectively. The electron density on the target plate increases up to around 600 mbar and then decreases with higher pressures. With the increase of the pressure, the electron temperature on the target decreases as shown in Fig. 5(e) and this may cause the density saturation on the target. On the other hand, the line-averaged density keeps increasing up to around $700 \mathrm{mbar}$ and then clearly decreases with 1000 mbar. The density on the target at 800 mbar is less than the peak density at $600 \mathrm{mbar}$ while the line-averaged density still keeps around the peak density even at 800 mbar. The density reduction rate of the line-averaged density after exceeding the peak density is less than that of the density on the target plate. We now have no information on electron temperature inside the target plates. However, the average electron temperature along the line of the interferometer chord passing through the center of plasma may be higher than that on the target, because in the upstream region temperature and density spatial distributions usually have their peak at the center. Therefore, the end loss plasma flux flowing into D-module may also have its peak stored energy at the center of the plasma. The difference in the pressure dependence of these densities may result from such a temperature difference.

\section{ACKNOWLEDGMENTS}

This work is performed with the support and under the auspices of the NIFS Collaboration Research program (Grant Nos. NIFS15KUGM102, NIFS14KUGM086, NIFS13KUGM078, and NIFS15KOAP029).

${ }^{1}$ H. Park et al., Rev. Sci. Instrum. 74, 4239 (2003).

${ }^{2}$ H. J. Hartfuss et al., Plasma Phys. Controlled Fusion 39, 1693 (1997).

${ }^{3}$ T. Yoshinaga et al., Plasma Fusion Res. 5, 030 (2010).

${ }^{4}$ N. Ito et al., Plasma Fusion Res. 10, 3402034 (2015).

${ }^{5}$ D. Kuwahara et al., J. Instrum. 10, C12031 (2015).

${ }^{6}$ Y. Nakashima et al., J. Nucl. Mater. 463, 537 (2015).

${ }^{7}$ Y. Nakashima et al., Trans. Fusion Sci. Technol. 63(1T), 100 (2013).

${ }^{8}$ X. Wang et al., Fusion Sci. Technol. 68, 178 (2015).

${ }^{9}$ J. Kohagura et al., J. Instrum. 10, C12024 (2015).

${ }^{10}$ Y. Nagayama et al., Rev. Sci. Instrum. 83, 10E305 (2012).

${ }^{11}$ D. Kuwahara et al., J. Plasma Fusion Res. Ser. 9, 125 (2010). 